\title{
ARTICLE
}

\section{Determination of current at a detector window using a hybrid adjoint function methodology}

\author{
Katherine Royston $^{\mathrm{a}^{*}}$, Alireza Haghighat ${ }^{\mathrm{a}}$, William Walters ${ }^{\mathrm{a}}, \mathrm{Ce} \mathrm{Yi}^{\mathrm{b}}$ and Glenn Sjoden ${ }^{\mathrm{b}}$ \\ ${ }^{a}$ Nuclear Engineering Program, Virginia Tech, Blacksburg, Virginia, USA; ${ }^{b}$ Nuclear and Radiological Engineering Program, \\ Georgia Tech, Atlanta, Georgia, USA
}

\begin{abstract}
A hybrid deterministic and Monte Carlo radiation transport methodology is developed for modeling an active interrogation system for detection of HEU in a container. This methodology includes four steps: i) neutron source transport and subcritical multiplication in cargo; ii) determination of neutron and gamma flux distributions due to fission neutrons throughout the cargo; iii) gamma-ray transport to the detector window; iv) detection of gamma-rays by the detector. This methodology enables accurate determination of neutron/gamma currents in real time for comparison with measurements, and examination of various material compositions. In this paper, we discuss the $3^{\text {rd }}$ step of the methodology, which quickly calculates an accurate gamma current at a detector window. An adjoint function formulation has been developed to determine the gamma current at the detector window by coupling the gamma distribution and pre-calculated adjoint function. To examine the new formulation, a model was created that is comprised of a standard cargo container, a D-T neutron source (14.1 $\mathrm{MeV}$ ), a sphere of HEU at the container center, and one-third density water cargo, which fills the container. To determine the adjoint function, we use the TITAN deterministic 3-D parallel $\mathrm{S}_{\mathrm{N}}$ code with the BUGLE-96 coupled neutron-gamma cross-section library. Our results are compared to Monte Carlo calculations using the MCNP5 code. Our method gives the gamma partial current at the detector window due to fission and so the MCNP5 reference solution is the difference between simulations with and without the HEU sphere. For the largest magnitude energy group, $2-3 \mathrm{MeV}$, with $1-\sigma$ statistical uncertainty of $\sim 11 \%$, a difference of $\sim 12 \%$ is observed between the hybrid and Monte Carlo predictions.
\end{abstract}

Keywords: radiation transport; deterministic; Monte Carlo; adjoint function; active interrogation

\section{Introduction}

A hybrid deterministic and Monte Carlo radiation transport methodology is developed for modeling an active interrogation system for detection of HEU in a cargo container. This methodology includes four steps: i) neutron source transport and subcritical multiplication in the cargo; ii) determination of neutron and gamma flux distributions due to fission neutrons throughout the cargo; iii) gamma transport to the detector window; iv) detection of gamma-rays by the detector. This methodology enables accurate determination of neutron/gamma currents in real time for comparison with measurements, and examination of various material compositions.

This paper discusses a novel adjoint particle transport formulation developed for the $3^{\text {rd }}$ step of the aforementioned hybrid methodology for determination of gamma current at the detector window by coupling the gamma distribution and pre-calculated adjoint functions. To demonstrate the methodology, we consider a cargo container with an HEU sphere, located inside, which is inspected using a detector-source assembly. To be able to perform real-time simulation of this interrogation system, we have developed a hybrid deterministic and Monte Carlo radiation transport methodology.

The paper is organized as follows. Section 2 derives a formulation for determining the angular current or partial current at a detector window based on the adjoint function. Section 3 describes the approach for analysis and benchmarking of the new methodology. Section 4 analyzes the results and considers the accuracy of the new hybrid formulation by comparing it with a reference Monte Carlo calculation. Further, the computation time of the new methodology is estimated and compared with the reference Monte Carlo calculation. Section 5 concludes the paper and recommends future work.

*Corresponding author. Email: royston@vt.edu 


\section{Derivation of flux/current formulation at the detector window}

To determine the angular gamma current at the detector window due to the gamma source distribution, we employ the following adjoint function methodology $[1,2]$. The forward transport equation is given by

$$
H \psi=S \text { in } \mathrm{V}
$$

where the transport operator $H$ is given as

$$
\begin{aligned}
H= & \hat{\Omega} \cdot \vec{\nabla}+\sigma(\vec{r}, E)- \\
& \int d E^{\prime} \int d \Omega^{\prime} \sigma_{s}\left(\vec{r}, E^{\prime} \rightarrow E, \hat{\Omega}^{\prime} \cdot \hat{\Omega}\right)
\end{aligned}
$$

For vacuum boundary conditions,

$$
\psi=0 \text { for } \hat{n} \cdot \hat{\Omega}<0 \text { on } \Gamma \text {. }
$$

The corresponding adjoint transport equation is given by

$$
H^{\dagger} \psi^{\dagger}=S^{\dagger} \text { in } \mathrm{V}
$$

where the adjoint transport operator $H^{\dagger}$ is given as

$$
\begin{aligned}
H^{\dagger}= & -\hat{\Omega} \cdot \vec{\nabla}+\sigma(\vec{r}, E)- \\
& \int d E^{\prime} \int d \Omega^{\prime} \sigma_{s}\left(\vec{r}, E \rightarrow E^{\prime}, \hat{\Omega} \cdot \hat{\Omega}^{\prime}\right)
\end{aligned}
$$

and we define an adjoint surface flux as

$$
\psi^{\dagger}=\tilde{\psi}^{\dagger} \text { for } \hat{n} \cdot \hat{\Omega}>0 \text { on } \Gamma \text {. }
$$

In the above equations, $S$ is the gamma source, $S^{\prime}$ is the adjoint source, $\Gamma$ refers to the surface bounding the problem volume $\mathrm{V}$, and $\psi$ and $\psi^{\dagger}$ are the gamma angular flux and angular adjoint function, respectively. Forming the commutation relation between Eq. (1) and Eq. (4):

$$
\left\langle\psi^{\dagger} H \psi\right\rangle-\left\langle\psi H^{\dagger} \psi^{\dagger}\right\rangle=\left\langle\psi^{\dagger} S\right\rangle-\left\langle\psi S^{\dagger}\right\rangle
$$

In Eq. (7), <> refers to integration over all independent variables. On the left-hand side of Eq. (7), only the streaming term of the transport operator, $\mathrm{H}$, will remain. On the right-hand side of Eq. (7), we have no adjoint source term $S^{\prime}$ and the commutation relation reduces to

$$
\begin{array}{r}
\int_{0}^{\infty} d E \int_{4 \pi} d \Omega \int_{V} d^{3} r \nabla \cdot \hat{\Omega} \psi(\vec{r}, E, \hat{\Omega}) \psi^{\dagger}(\vec{r}, E, \hat{\Omega}) \\
=\left\langle\psi^{\dagger} S\right\rangle
\end{array}
$$

Using the divergence theorem, we can then expand the left-hand side in Eq. (8) into two integrals over $\Omega$.

$$
\begin{aligned}
\int_{0}^{\infty} d E & \int_{\hat{n} \cdot \hat{\Omega}>0} d \Omega \int d \Gamma \hat{n} \cdot \hat{\Omega} \psi \psi^{\dagger}+ \\
& \int_{0}^{\infty} d E \int_{\hat{n} \cdot \hat{\Omega}<0} d \Omega \int d \Gamma \hat{n} \cdot \hat{\Omega} \psi \psi^{\dagger}=\left\langle\psi^{\dagger} S\right\rangle
\end{aligned}
$$

Given the chosen container model, the adjoint source is zero, but there is an outgoing boundary adjoint flux from the container as defined in Eq. (6). This gives Eq. (10).

$$
\begin{array}{r}
\int_{0}^{\infty} d E \int d \Gamma \int_{\hat{n} \cdot \hat{\Omega}>0} d \Omega \hat{n} \cdot \hat{\Omega} \psi(\vec{r}, E, \hat{\Omega}) \psi^{\dagger}(\vec{r}, E, \hat{\Omega}) \\
=\left\langle\psi^{\dagger} S\right\rangle
\end{array}
$$

Considering a detector that is sensitive to one energy, one position, and one direction, the boundary adjoint flux is given by

$$
\tilde{\psi}^{\dagger}=\delta\left(E-E_{g}\right) \delta\left(\vec{r}-\vec{r}_{d}\right) \delta\left(\hat{\Omega} \cdot \hat{\Omega}_{n}\right)
$$

With Eq. (11), Eq. (10) can be solved for the angular current at the detector window as

$$
J\left(\vec{r}_{d}, E_{g}, \hat{\Omega}_{n}\right)=\left\langle\psi^{\dagger} S\right\rangle
$$

In Eq. (12), the adjoint function $\psi^{\dagger}$ is found by solving Eq. (4) and the gamma source $S$ is obtained from steps i and ii [3] as discussed in Section 1. Note that to obtain the partial current in this paper, we utilize the following expression for the adjoint flux:

$$
\tilde{\psi}^{\dagger}=\delta\left(E-E_{g}\right) \delta\left(\vec{r}-\vec{r}_{d}\right)
$$

\section{Methodology}

\subsection{Description of the reference model}

The reference case chosen to test the methodology is active interrogation of a cargo container with HEU inside. A D-T source $(14.1 \mathrm{MeV})$ is represented as an isotropic surface source of size $13.5 \times 13.5 \mathrm{~cm}^{2}$. The detector window is represented as a surface of size $13.5 \times 13.5 \mathrm{~cm}^{2}$. Figure 1 depicts the model geometry.

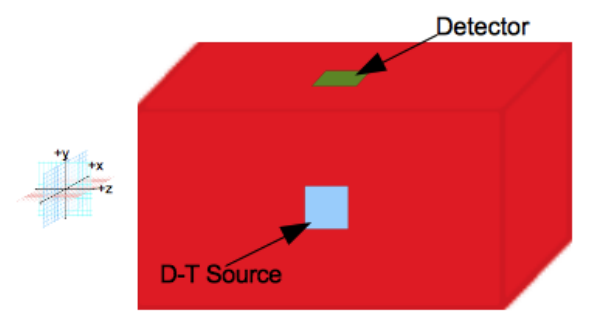

Figure 1. Reference cargo container model in an active interrogation environment. 
Not shown in Figure 1 is the SNM material, i.e., HEU, which is placed at the center of the container as a sphere of radius $6.75 \mathrm{~cm}$. This size sphere corresponds to $25 \mathrm{~kg}$ of HEU. For this model, the container is made of $1 \mathrm{~cm}$ thick steel and is filled with water of one-third nominal density. The size of the reference cargo container is $8 \mathrm{ft}$ $(2.4384 \mathrm{~m}) \times 8.5 \mathrm{ft}(2.5908 \mathrm{~m}) \times 20 \mathrm{ft}(6.096 \mathrm{~m})$.

\subsection{Computer codes}

\subsubsection{TITAN deterministic transport code}

The TITAN code is a hybrid deterministic transport code $[4,5]$, which was used to solve for the adjoint gamma function in Eq. (4). In addition to quadrature order, the TITAN code allows the user to "split" quadrature set directions in a technique called ordinate splitting (OS) [6]. OS allows additional directions to be created along important directions and also for directions closer to the axes to be created without increasing the quadrature order. One of the quadrature sets used in this work is given in Figure 2.

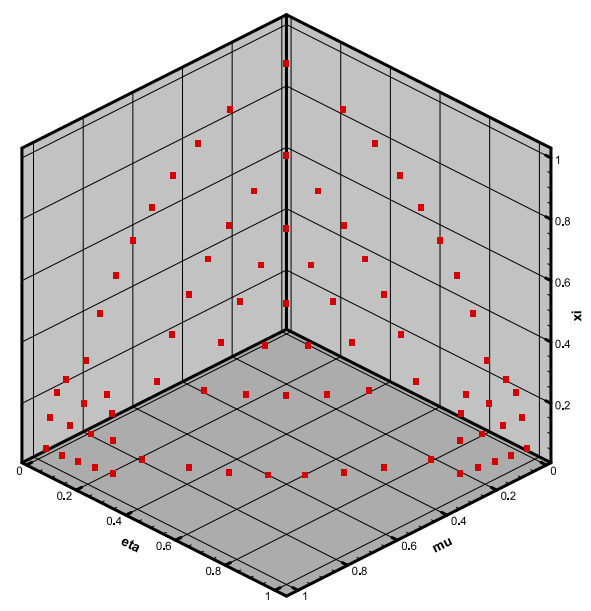

Figure 2. TITAN Level Symmetric $\mathrm{S}_{20}$ quadrature with ordinate splitting along $\mathrm{x}$ - and $\mathrm{y}$-axes.

In Figure 2, we see the level symmetric $\mathrm{S}_{20}$ quadrature with OS of the directions closest to the $\mathrm{x}$ - and $\mathrm{y}$-axes. The $\mathrm{S}_{20}$ quadrature has 55 directions per octant, but each split direction adds 13 more per octant in this example. Splitting along the $\mathrm{y}$-axis was chosen to provide additional directions from the HEU region to the detector and a direction that is closer to the y-axis, which could be used to represent a collimated detector.

\subsubsection{MCNP5 Monte Carlo code}

The MCNP5 Monte Carlo code [7] was used as a reference solution to compare the hybrid method with. The MCNP5 model records current tallies on the detector surface. To determine the current at the detector window only from the HEU, two calculations are needed. First, the current for the model with the HEU present is found. Second, the current is calculated with no HEU in the model to give us the contribution from the neutron source. The difference between these currents gives the current solely due to the HEU.
Cross sections are an important difference between the new hybrid formulation and the MCNP5 benchmark. The MCNP5 code uses continuous energy cross sections, while steps $i i$ and iii use discrete energy groups. The MCNP5 solution uncertainty must also be considered. Uncertainty propagation is used to determine the uncertainty of the difference between the two MCNP5 calculations. Because the difference between the two calculations is small, the uncertainty will be much larger than the uncertainties of the original calculations.

\subsection{Cross-section data}

Multi-group cross sections are needed for steps $i i$ and iii of the hybrid methodology. The 67-group BUGLE-96 neutron-gamma coupled cross-section data is used [8]. The BUGLE-96 cross sections contain 47 neutron groups and 20 gamma groups.

\subsection{Development of an effective calculation model}

Recalling that the HEU sphere is placed at the center of the container, we may reduce the model size by considering the detector field-of-view (FOV) along the $\mathrm{z}$-axis (container length). To develop this effective calculation model, we have performed a series of calculations in the TITAN code to determine the FOV of the detector window.

The detector response can be found using the adjoint function coupled with the gamma source distribution as

$$
R=\sum_{i} \sum_{g} \phi_{i, g}^{\dagger} S_{i, g} V_{i}
$$

The fractional contribution of each spatial cell to the detector response is given by Eq. (15) [9].

$$
C=\sum_{g} \phi_{i, g}^{\dagger} S_{i, g} V_{i} / R
$$

To determine an appropriate shorter container length, the detector response contribution was summed along the $\mathrm{z}$-axis outward from the container center (HEU location), as depicted in Figure 3. A container length of $140.768 \mathrm{~cm}(-70.384 \mathrm{~cm}$ to $+70.384 \mathrm{~cm})$ was chosen and reduced the model volume by nearly $77 \%$, while only reducing the calculated detector response by $3.9 \%$.

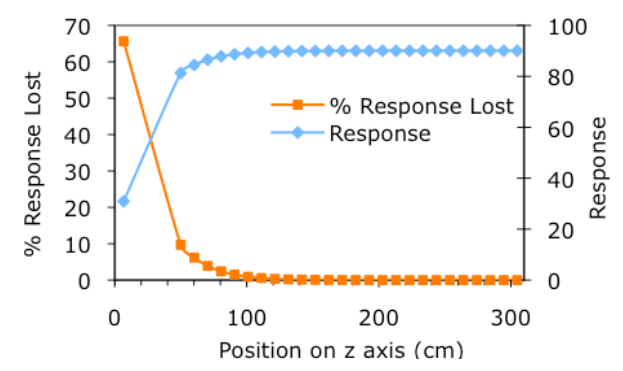

Figure 3. Detector response and response lost (\%) versus distance from container center along $z$-axis included in response calculation. 


\section{Results and analysis}

As discussed in Section 1, our hybrid formulation calculates the current at the detector window due to the HEU using a fast calculation of the neutrons from subcritical multiplication and a pre-calculated adjoint function. The adjoint function must be pre-calculated for each gamma energy group that is needed.

We desire to benchmark the hybrid methodology by comparison with the MCNP5 Monte Carlo code. The gamma current at the detector window is found using MCNP5 as described in Section 3.2.2. We will compare results for gamma groups 8-16 (0.1-3.0 MeV) because other energy groups do not have a significant contribution to the current from the HEU. We will consider different parameters for our hybrid method, including scattering order $\left(\mathrm{P}_{1}, \mathrm{P}_{3}\right.$, and $\left.\mathrm{P}_{5}\right)$, quadrature $\left(\mathrm{S}_{20}, \mathrm{~S}_{30}\right.$, and $\left.\mathrm{S}_{36}\right)$, and mesh refinement. Quadrature orders higher than 20 use the Legendre-Chebyshev quadrature set [10] instead of the level-symmetric quadrature set. For mesh refinement, the initial meshing is referred to as the "base meshing." "Refine 1" will refer to a mesh where the number of meshes has been doubled in each spatial direction for the coarse meshes between the HEU sphere and the detector window. In "Refine 2", the meshing between the HEU and detector is doubled again in each direction.

The gamma current at the detector window for the energy groups of interest using the hybrid method with increasing anisotropic scattering order is given in Figure 4. The horizontal axis gives the mid-point of each energy group. The difference in $\mathrm{P}_{1}$ and $\mathrm{P}_{3}$ relative to $\mathrm{P}_{5}$ is given in Figure 5.

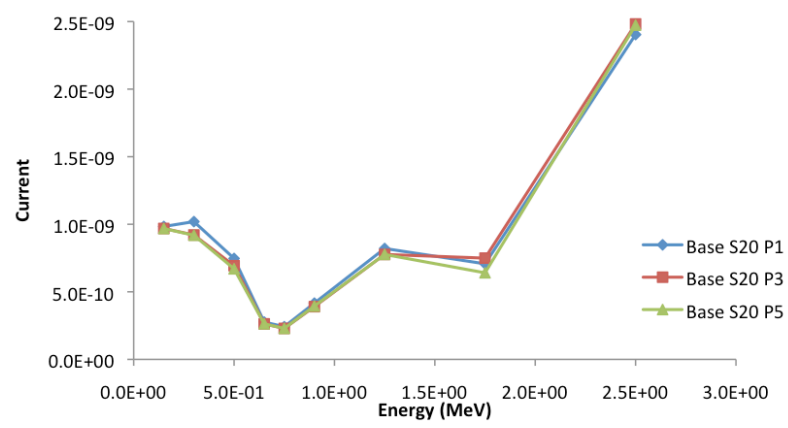

Figure 4. Hybrid method current spectra at detector window due to HEU in cargo container with increasing scattering order.

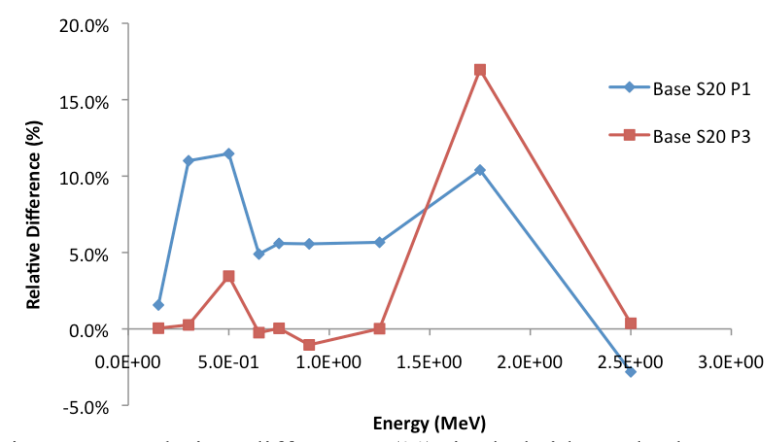

Figure 5. Relative difference $(\%)$ in hybrid method current spectra due to HEU for $\mathrm{P}_{1}$ and $\mathrm{P}_{3}$ relative to $\mathrm{P}_{5}$.
In Figure 4, the adjoint model uses base meshing with $\mathrm{S}_{20}$ quadrature. While some differences are visible, Figure 5 gives a better indication of magnitudes. From Figure 5, significant differences are seen with increasing anisotropy order and it is determined that $\mathrm{P}_{5}$ is necessary. All following simulations use $\mathrm{P}_{5}$ scattering order and the generation of cross-sections with higher scattering orders is being considered.

Next, mesh refinement is considered. The current spectra for the base, refine 1 , and refine 2 meshes are given in Figure 6.

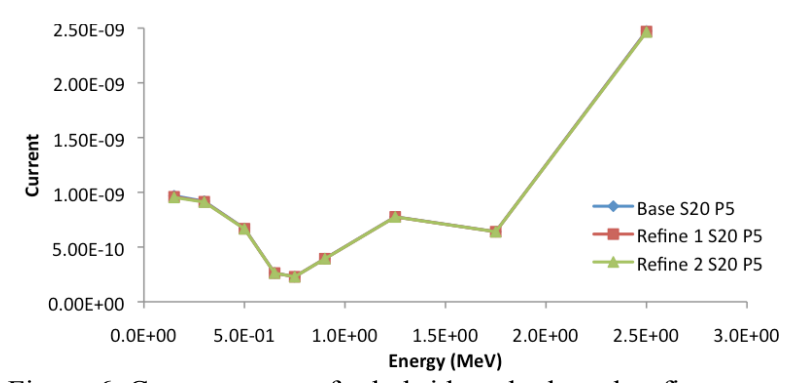

Figure 6. Current spectra for hybrid method mesh refinement.

In Figure 6, it is clear that the base meshing is sufficient and mesh refinement does not alter the hybrid method result. The hybrid method is now considered with different quadrature orders. In Figure 7, the hybrid method uses the base meshing and $\mathrm{P}_{5}$ scattering order.

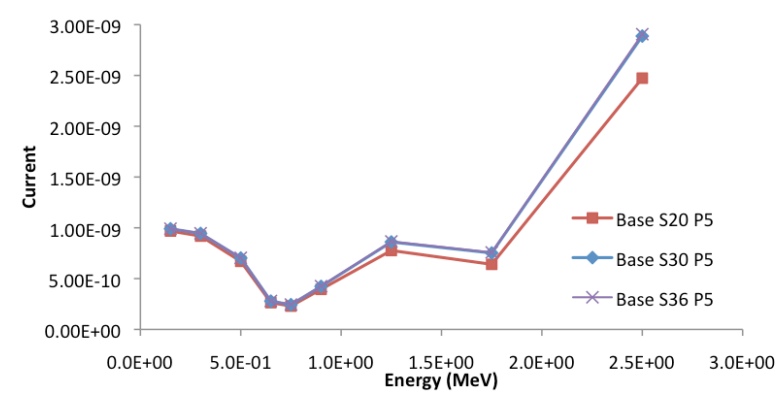

Figure 7. Current spectra for the hybrid method with $\mathrm{S}_{20}, \mathrm{~S}_{30}$, and $\mathrm{S}_{36}$ quadratures.

In Figure 7, the higher energies show a significant increase in current with the quadrature increase from $S_{20}$ to $\mathrm{S}_{30}$. The $\mathrm{S}_{20}$ quadrature has 55 directions per octant (81 with splitting), while the $S_{30}$ quadrature has 120 (146 with splitting). Figure 7 indicates that the higher number of directions in $S_{30}$ is necessary to solve the problem, while $\mathrm{S}_{36}$ shows no further improvement.

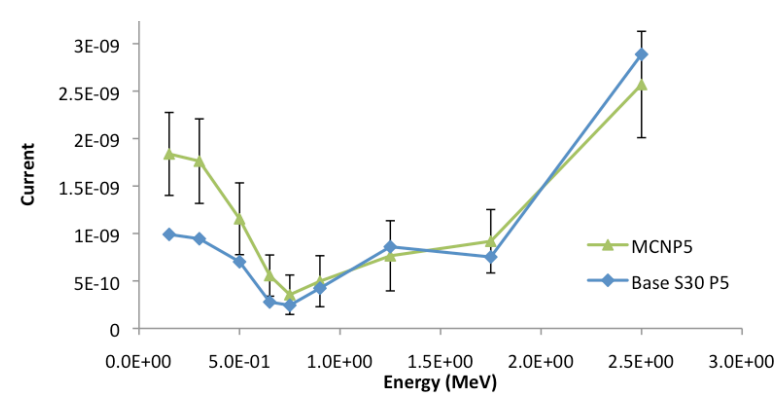

Figure 8. Current spectra for MCNP5 ( $2 \sigma$ error bars) and hybrid method (base meshing, $\mathrm{P}_{5}$ and $\mathrm{S}_{30}$ ). 
Figure 8 compares the hybrid method with MCNP5 predictions. The energy group with the largest response (2-3 MeV) gives a relative difference of $12.4 \%$ between the hybrid method and MCNP5 ( $1 \sigma$ uncertainty of $10.9 \%$ ). However, the MCNP5 values given in Figure 8 clearly have large uncertainties and therefore are not precise. As discussed previously, taking the difference between two close numbers results in a much larger uncertainty. Here, the nature of our problem limits the use of variance reduction techniques in the MCNP5 code, because the problem has two main objectives: i) determination of fission density at the HEU; ii) generation of gamma rays and their transport to the detector. These are two independent processes with diverse objectives.

Potential causes of differences between the hybrid method and MCNP5 include the cross sections used and approximations in step $i$ [3] of the hybrid methodology. Differences in cross sections have already been discussed. A fast response-function methodology is used in step $i$ to calculate fission in the HEU [3], which may result in some differences in the gamma source distribution that is coupled with the adjoint function.

The computation times of the hybrid method and MCNP5 were compared. In Table 1, the computation times for some pre-calculated values for the hybrid method are given. The adjoint function computation time will depend on the energy group of interest so the shortest $(2-3 \mathrm{MeV})$ and the longest $(100-200 \mathrm{keV})$ times are given. The adjoint function calculation times are for the base meshing with $\mathrm{S}_{30}$ and $\mathrm{P}_{5}$ run on 8 processors.

Table 1. Computation times for pre-calculated values in the hybrid methodology. Adjoint calculations are on 8 processors.

\begin{tabular}{lc}
\hline Pre-calculated Values & Time $(\min )$ \\
\hline Response Coefficients & 2400 \\
Adjoint Group $8(2-3 \mathrm{MeV})$ & 136 \\
Adjoint Group 16 $(100-200 \mathrm{keV})$ & 349 \\
\hline
\end{tabular}

In Table 2, the computation times to find the gamma current at the detector window for nine energy groups of interest for the hybrid methodology and MCNP5 are given. MCNP5 parallel calculations are on 8 processors.

Table 2. Gamma current computation times for the hybrid methodology and MCNP5 for nine energy groups.

\begin{tabular}{cc}
\hline Method & Time $(\mathrm{min})$ \\
\hline MCNP5 & 11704 \\
Hybrid & 9.6 \\
\hline
\end{tabular}

In Table 1, it is clear that the pre-calculated hybrid method values do require a reasonable length of time to prepare. Using the pre-calculated values, the hybrid method takes only 9.6 minutes to find the gamma current for the nine energy groups. In contrast, the MCNP5 results take over 1200 times longer and still have large uncertainties. For all nine energy groups to have $1 \sigma$ uncertainties less than $10 \%$, we estimate from the figure of merit that the MCNP5 code would have to run for more than 79 days on 8 processors.

\section{Conclusion}

In conclusion, we have developed an adjoint transport formulation for determination of the gamma current at a detector window for a given neutron/gamma distribution. It is demonstrated that for the 2-3 MeV gamma energy group, for which MCNP5 has the lowest uncertainty, the results of the new formulation are agree with MCNP5. We are investigating the use of higher scattering order cross-sections to see if more improvements can be made. Timing limitations with the MCNP5 model made the comparison with the hybrid method difficult. The long computation time for MCNP5 further proves the need for the new formulation developed in this study.

\section{Acknowledgements}

This work was funded by the U.S. National Nuclear Security Administration (NNSA).

\section{References}

[1] E. Lewis and W. Miller, Computational Methods of Neutron Transport, John Wiley \& Sons, New York, (1984).

[2] A. Haghighat and G. Sjoden, 3-D particle transport methods and their applications, Applied Modeling and Computations in Nuclear Science, T. M. Semkow, S. Pommé, and S. M. Jerome, Eds., American Chemical Society, Washington, D. C., (2006), pp. 162-182.

[3] W. Walters, A. Haghighat, K. Royston, C. Yi and G. Sjoden, A response-function methodology to calculate induce fission neutron flux distribution in an active interrogation system, Proc. ICRS-12 \& RPSD-2012, Nara, Japan, Sept. 2-7, 2012, (2012).

[4] C. Yi, Hybrid discrete ordinates and characteristics method for solving the Linear Boltzmann Equation, PhD Thesis, University of Florida, (2007).

[5] C. Yi and A. Haghighat, A 3-D block-oriented hybrid discrete ordinates and characteristics method, Nucl. Sci. Eng. 164 (2010), pp. 221-247.

[6] G. Longoni and A. Haghighat, Development of the regional angular refinement and its application to the CT-scan device, Trans. Am. Nucl. Soc., 86 (2002), pp. 246-248.

[7] X-5 Monte Carlo Team, MCNP-A General Monte Carlo Code for Neutron and Photon Transport, Version 5, Los Alamos National Laboratory, (2003).

[8] Bugle-96: Coupled 47 Neutron, 20 Gamma-Ray Group Cross Section Library Derived from ENDF/B-VI for LWR Shielding and Pressure Vessel Dosimetry Applications, DLC-76, Oak Ridge National Laboratory, (1996).

[9] G. I. Bell and S. Glasstone, Nuclear Reactor Theory, Robert E. Krieger Publishing, Malabar, FL (1985).

[10] B. G. Carlson, Transport Theory: Discrete Ordinates Quadrature over the Unit Sphere, LA-4554, Los Alamos National Laboratory, (1970). 